\title{
A Double-Blind Placebo-Controlled, Randomized Pilot Study: Consump- tion of a High-Metabolite Immunogen from Yeast Culture has Beneficial Effects on Erythrocyte Health and Mucosal Immune Protection in Healthy Subjects
}

\author{
Gitte S. Jensen*,a, Kelly M. Patterson ${ }^{\mathrm{b}}$, Janelle Barnes ${ }^{\mathrm{b}}$, Alexander G. Schauss ${ }^{\mathrm{c}}$, Robert Beaman ${ }^{\mathrm{d}}$, \\ Stuart G. Reeves ${ }^{\mathrm{e}}$ and Larry E. Robinson ${ }^{\mathrm{e}}$ \\ ${ }^{a}$ Holger NIS Inc., $60113^{\text {th }}$ Avenue NE, Calgary Alberta Canada T2E 1C7 \\ ${ }^{b}$ NIS Labs, 1437 Esplanade, Klamath Falls OR 97601 \\ ${ }^{c}$ Natural and Medicinal Products Division, AIBMR Life Sciences, 4117 South Meridian, Puyallup WA 98373, U.S.A. \\ ${ }^{d}$ Beaman's Wellness Center, 1903B Austin St., Klamath Falls OR 97603 \\ ${ }^{e}$ Embria Health Sciences, 2105 SE Creekview Drive, Ankeny, IA 50021
}

\begin{abstract}
This double-blind, randomized, placebo-controlled pilot study was designed to evaluate effects of consumption of an antioxidant-rich, yeast culture-based high-metabolite immunogen EpiCor ${ }^{\mathbb{B}}$. Twenty-five healthy participants consumed 0.5 gram EpiCor ${ }^{\circledR}$ or placebo daily for 5 weeks. The hematocrit increased significantly in the EpiCor ${ }^{\circledR}$ group $(p<0.04)$. A mild increase in saliva sIgA upon EpiCor ${ }^{\mathbb{B}}$ consumption $(p=0.16)$ prompted a subsequent 8 -week open-label study involving 22 people showing significant increase in $\operatorname{sIgA}(p<0.05)$. EpiCor ${ }^{\circledR}$ consumption led to a mild increase in serum IL-10 $(p<0.2)$; no other differences in Th1/Th2 cytokines were observed. Minor health complaints decreased in the EpiCor $^{\mathbb{B}}$ group compared to the placebo group $(p<0.02)$. Seasonal allergies increased in the placebo group, but were not observed in the EpiCor ${ }^{\mathbb{B}}$ group; this was reflected by increased serum IgE in the placebo group compared to the EpiCor ${ }^{\mathbb{B}}$ group $(p<0.13)$. We conclude that consumption of EpiCor ${ }^{\mathbb{R}}$ supported the health of red blood cells and mucosal immune protection.
\end{abstract}

Keywords: Erythrocyte, hematocrit, allergy, IgE, immune, Th1/Th2, inflammation, sIgA.

\section{INTRODUCTION}

EpiCor $^{\circledR}$ is a high-metabolite immunogen based on baker's yeast (Saccharomyces cerevisiae), and is a natural fermentation product based on culture under anaerobic conditions and stress. The complex profile of bioactive components includes the nutrient/vitamin profile, yeast cell wall components, as well as stress-induced metabolites and other components from the proprietary medium. Even though many yeast cell wall compounds, such as beta-glucans and mannans, are predominantly pro-inflammatory, this yeastbased product is more complex and contains high levels of antioxidants [1-3], which are available to cells and protect them from oxidative damage in vitro [3].

The trend in industrialized countries is towards an increasingly older population, and the interest in preventive health strategies, including the effects of antioxidant-rich, immunomodulatory foods and supplements is growing rapidly. Meanwhile, meta-studies on antioxidants in cardiovascular disease and cancer have focused primarily on single antioxidant vitamins and remain only partially conclusive, presumably as multiple parameters involved in oxidative

*Address correspondence to this author at Holger NIS Inc., $60113^{\text {th }}$ Avenue NE, Calgary Alberta Canada T2E 1C7; Tel: (403) 277-4134;

E-mail: gitte@holgernis.com stress may vary between individual studies, including selection of study population, exposure to smoke and pollution, level of exercise, cultural background as it pertains to diet, and psychological stress. The detrimental effects of reduced intake of antioxidants are linked to the inflammatory conditions associated with obesity [4], as well as age-related cognitive decline $[5,6]$. Furthermore, low serum levels of antioxidant compounds have recently been correlated to other behavioral disturbances such as attempted suicide [7]. Thus, the importance of antioxidant intake is relevant for many areas of preventive medicine and health.

Previous studies on immunomodulatory effects of consumption of complex Saccharomyces cerevisiae-containing nutritional products have shown increased survival and daily activity in a murine model of Chronic Fatigue Syndrome [8] and in a porcine model of severe inflammation [9].

An in vitro study was performed to assess the antiinflammatory potential and direct effects of EpiCor ${ }^{\circledR}$ on different lymphocyte subsets and to identify mechanisms of action. The in vitro study showed that EpiCor ${ }^{\circledR}$ has a high anti-oxidant capacity in a cell-based system, and specifically activates human NK and B cells in vitro [3].

The present study was based on the findings from previous human studies $[1,2]$ and our recent in vitro study with EpiCor $^{\mathbb{B}}[3]$. It was designed as a pilot study to evaluate pos- 
sible effects of EpiCor ${ }^{\circledR}$ consumption on saliva and serum immunoglobulins, Th1/Th2 cytokine levels, and level of inflammation.

\section{MATERIALS AND METHODS}

\section{Double Blind Placebo Controlled Study Design}

This study was designed as a pilot study for an initial evaluation of the effects of EpiCor ${ }^{B}$ consumption on general health. A double-blinded randomized placebo-controlled study design was used. Twenty-six people were recruited, and represented 13 men and 13 women of similar age range. Thirteen subjects (6 males and 7 females) were randomized for placebo consumption, and 13 subjects $(7$ males and 6 females) were randomized for EpiCor $^{\circledR}$ consumption. One male in the placebo group was lost to follow-up. At study entry (T0), and after 5 weeks of consumption (T35), the following samples were collected from each subject: One saliva collection tube with $2-4 \mathrm{ml}$ unstimulated saliva for secretory $\operatorname{IgA}(\mathrm{sIg}$ ) measurement; one EDTA tube for performing a complete blood count (CBC) with differential; and two serum separator tubes for collecting sufficient aliquots of serum for total $\operatorname{IgA}, \operatorname{IgG}$, IgE, and Th1/Th2 cytokine profile.

\section{Consumables}

Two consumables were tested at a dose of 0.5 gram/day: EpiCor $^{\circledR}$ and placebo, both encapsulated with 0.5 gram/capsule in veggie caps. EpiCor ${ }^{\circledR}$ was tested for antioxidant capacity using the Oxygen Radical Absorbance Capacity (ORAC) test (Brunswick Laboratories, Norton, MA), and had an ORAC value of 450-650 micromolar Trolox Equivalents per gram. The placebo was made from finely ground brown rice flour mixed with $1 \%$ molasses and $1 \%$ tamari (soy sauce). Five hundred capsules each of EpiCor ${ }^{\mathbb{R}}$ and placebo were received in bulk at NIS Labs. The capsules were labeled with codes to ensure that all investigators, nurses, and staff at the clinic and lab were conducting the study, interacting with study participants, and analyzing data in a blinded fashion. At NIS Labs, five pill boxes, each containing supplies for one week, were prepared for each study participant. Thirteen sets of EpiCor ${ }^{\mathbb{B}}$ and placebo were prepared.

\section{Study Participants and Compliance}

Twenty-six people (13 females and 13 males) were recruited and scheduled for a physical exam with a medical doctor. Upon passing the exam, each person was randomized to either group EpiCor ${ }^{\mathbb{B}}$ or placebo. Males and females were randomized separately, to ensure an even gender distribution in each group. The initial saliva and blood samples (T0) were collected, and instructions were given regarding consumption, tracking, and compliance. A calendar was provided for notes to record sickness, major life events, and stressors. After five weeks of consumption, volunteers were instructed to return with the pill boxes and the calendar. Both were used in parallel for tracking compliance, as the subjects had been instructed to leave any missed capsules in the pill boxes. The next saliva and blood samples were collected upon completion of the 5 week ( $=35$ days) consumption period (T35). Questionnaires pertaining to general health were also completed 1 and 2 weeks after consumption had ended.

One study participant enrolled but did not return for follow-up visits. The remaining 25 participants completed the study. The age and gender distribution, as well as the height, weight, and body mass index (BMI) of these study participants is shown in Table 1.

The randomization led to an overall comparable gender and age distribution; however, the males in the placebo group were younger than the males in the EpiCor ${ }^{\mathbb{B}}$ group. Despite one male in the placebo group with a Body Mass Index (BMI) of 42, the average BMI was comparable between the two groups. Sixteen of 25 people (64\%) had 100\% compliance. Of the remaining participants, three missed a single capsule, two missed two capsules, two missed three capsules, and two missed four capsules. The average compliance was $98+/-0.04 \%$.

\section{Open-Label Study of Saliva sIgA}

As a follow up study to confirm the Salivary IgA results, an open label study was conducted. Twenty-two naïve study participants were recruited and monitored for SIgA changes over a longer period of time, for a total of 12 weeks. The first 4 weeks served as a baseline before EpiCor ${ }^{\mathbb{B}}$ consumption; thereafter EpiCor ${ }^{\circledR}$ was consumed for the next 8 weeks. Saliva samples were collected morning and evening (pooled) three days per week throughout the 12 weeks. All individual samples were analyzed; the results for each individual for each 4 week period were pooled, and the three 4 week periods were compared for statistical significance.

Table 1. Age/gender Distribution and Body Mass Index (BMI) of Study Participants in the Two Treatment Groups EpiCor ${ }^{\circledR}$ and Placebo

\begin{tabular}{|c|c|c|c|c|}
\hline & \multicolumn{2}{|c|}{ EpiCor } & \multicolumn{2}{c|}{ Placebo } \\
\hline Age range & \multicolumn{2}{|c|}{$\mathbf{1 9 - 4 3}$ years } & \multicolumn{2}{c|}{$\mathbf{2 5 . 5} \pm \mathbf{8 . 0}$} \\
\hline Mean Age & \multicolumn{2}{|c|}{$\mathbf{2 9 . 7 \pm 9 . 5}$} & Males & Females \\
\hline \hline Males & 6 & 7 & $6 *$ & $164.3 \pm 9.5$ \\
\hline Average height (cm) & $178.5 \pm 8.2$ & $162.8 \pm 4.0$ & $92.4 \pm 34.6$ & $72.3 \pm 15.0$ \\
\hline Average weight (kg) & $88.7 \pm 13.5$ & $62.5 \pm 8.2$ & $26.8 \pm 8.0$ & $26.6 \pm 2.2$ \\
\hline Average BMI & $27.8 \pm 2.9$ & $23.5 \pm 2.0$ & \\
\hline
\end{tabular}

*Seven males were enrolled. One was lost to follow up. 


\section{Subjective Health Assessment}

Two tools were used for collecting subjective feedback on the effects of consumption: a) an open question: "Did you feel any changes in your health that you believe were related to consuming the capsules? If so, please describe", and b) a list of 22 questions pertaining to minor health issues, such as poor gums, headaches, heartburn, constipation, and irritated skin. Each question was answered either no ( 0 points), mild ( 1 point), moderate ( 2 points), or severe ( 3 points), and the questionnaires were scored by adding the number of points per person/time point. Both sets of questionnaires were analyzed separately for EpiCor versus placebo, and the overall change between the two groups was compared.

\section{Complete Blood Count (CBC)}

The blood samples collected into EDTA vials were used for triplicate $\mathrm{CBC}$ measurements with differential using a Coulter Micro Diff II machine (Beckman Coulter, Hialeah FL). The triplicate data sets were averaged for each person/time point.

\section{Saliva IgA}

The collected saliva was stored at $4^{\circ} \mathrm{C}$ immediately upon collection, and frozen at $-20^{\circ} \mathrm{C}$. Saliva IgA was quantified using a commercial ELISA kit from Salimetrics LLC (State College PA). All samples were assayed in duplicate at a 1:100 dilution in assay buffer, according to instructions from the manufacturer.

\section{Serum Immunoglobulins IgA, IgG, and IgE}

Serum IgA, IgG, and IgE were quantified using commercial ELISA kits from Bethyl Laboratories Inc. (Montgomery $\mathrm{TX}$ ). Serum $\operatorname{IgA}, \operatorname{IgE}$, and $\operatorname{IgG}$ was assayed initially at $1: 10,000$ fold dilution, and samples that did not fall on the standard curve were re-assayed at dilutions up to $10^{6}$.

\section{High-Sensitivity C-Reactive Protein (CRP)}

The levels of serum CRP were determined using an ELISA kit from Alpha Diagnostic International (San Antonio TX). All samples were assayed in duplicate at 100 -fold dilutions in assay buffer, following the instructions from the manufacturer.

\section{Th1/Th2 Cytokine Bead Array}

The Th1/Th2 cytokine profile was evaluated using a cytokine bead array (Becton-Dickinson, San Jose, CA) which measures the relative amounts of the following 6 cytokines: Interleukin-2 (IL2), Interleukin-4 (IL4), Interleukin-6 (IL6), Interleukin-10 (IL10), Tumor Necrosis Factor-alpha (TNF $\alpha$ ), and Interferon-gamma (IFN $\gamma$ ). Samples were acquired on a FacsCalibur flow cytometer (Becton-Dickinson, San Jose, CA) using the CellQuest Pro software (Becton-Dickinson, San Jose, CA), with 20,000 events saved per sample. Data analysis was performed using the FlowJo software (TreeStar Inc., Ashland, OR). Electronic gating was performed to exclude clumps of beads and include single beads only. Subsequently, the fluorescence intensity in FL3 was used to electronically gate for each bead type specific for each cytokine. For each bead type, the fluorescence intensity in FL1 was proportional to the amount of cytokine captured by the beads, and thus proportional to the amount of this cytokine in the serum samples. The mean fluorescence intensities were exported to Excel for calculation of mean and standard deviation, and to perform statistical analysis of differences over time.

\section{Cell Proliferation}

The Daudi B cell leukemia line (American Type Culture Collection, Manassas, VA) was cultured in log phase in culture medium as suggested by the supplier. Cells were stained with the lipophilic membrane dye PKH67 (Sigma Aldrich St. Louis, MO), and cultured for 48 hours in the absence versus presence of serial dilutions of EpiCor ${ }^{\mathbb{B}}$. EpiCor ${ }^{\mathbb{B}}$ was prepared for addition to cell culture as previously described in detail [3]. In brief, $0.5 \mathrm{~g}$ EpiCor $^{\circledR}$ was added to $5 \mathrm{~mL}$ phosphate-buffered saline, mixed well, and incubated at room temperature for 1 hour. A $10 \%$ solution was made in RPMI culture medium, and incubated at $37^{\circ} \mathrm{C}$ for 1 hour. Solids were precipitated by centrifugation, and the liquid phase was sterile-filtered using a 0.2 micron cellulose-acetate filter. Serial dilutions of the sterile liquid phase were prepared in culture medium. After the 48 hours incubation, the cells were washed and transferred to vials for flow cytometry to examine growth-induced loss of fluorescence intensity as a result of daughter cells sharing the membrane fluorescence of the mother cell. The samples were analyzed by flow cytometry using a FacsCalibur flow cytometer (Becton Dickinson San Jose, CA) and FlowJo software (TreeStar Ashland, $\mathrm{OR})$. The proportion of cells with reduced fluorescence intensity, compared to the freshly stained cells before culturing was used to evaluate the proportion of daughter cells, as a measure of proliferative activity in the cultures.

\section{Programmed Cell Death (Apoptosis)}

The Daudi B cell leukemia line (American Type Culture Collection, Manassas, VA) and peripheral blood mononuclear cells (PBMC) were cultured for 48 hours in the absence versus presence of serial dilutions of EpiCor ${ }^{\circledR}$. Untreated cultures were tested in quadruplicate, and each serial dilution of EpiCor ${ }^{\circledR}$ was tested in duplicate. The experiment was repeated three times. After the 48-hour incubation, cells were washed and transferred into Annexin Binding Buffer with AnnexinV (Molecular Probes (Eugene, OR) and 7AAD (Beckman Coulter, Miami, FL). Cells that stain with both AnnexinV and 7AAD are known to represent cells in an advanced and irreversible state of apoptosis. The samples were analyzed by flow cytometry using a FacsCalibur flow cytometer (Becton Dickinson San Jose, CA) and FlowJo software (TreeStar Ashland, OR). The percent cells positive for AnnexinV and 7AAD was analyzed as a measure of apoptotic cells in the cultures.

\section{Statistical Analysis}

Comparison of arithmetic means was performed using Student's t-test, using Microsoft Excel (Microsoft Corporation, Redmond, WA). Probabilities ( $p$ ) were computed as the tail area of the $t$ distribution using a two-tailed t-test. A 5\% level of significance (i.e. chance of error) was chosen. If $p<0.05$, the data were judged to be statistically significant. For the analysis of saliva and serum immunoglobulins, where the levels across the study population were highly variable but remain fairly constant within each person, the normalized data for each person/time after consumption was 
Table 2. Changes in Erythrocyte Numbers and Quality as a Result of Consumption of EpiCor ${ }^{\circledR}$ Versus Placebo

\begin{tabular}{|c|c|c|c|c|}
\hline & EpiCor & & \multicolumn{2}{c|}{ Placebo } \\
\hline & T0 & T35 & T0 & $5.3 \pm 0.5$ \\
\hline \hline Erythrocytes $10 \% / \mathrm{mL}$ & $4.9 \pm 0.5$ & $4.9 \pm 0.4$ & $14.8 \pm 1.5$ & $14.6 \pm 1.6$ \\
\hline Hemoglobin (g/dL) & $13.7 \pm 1.3$ & $13.9 \pm 1.1$ & $43.0 \pm 3.5$ & $43.2 \pm 6.1$ \\
\hline
\end{tabular}

*The increase in hematocrit after 5 weeks of consumption of EpiCor ${ }^{\mathbb{B}}$ was statistically significant $(p<0.04)$.

subtracted from the same data set before consumption. Statistical significance was tested using Student's t-test. Statements about whether a given mean value of normalized data was different from a reference mean value were evaluated by two-tailed independent t- tests and were supported if $p<0.05$. If $p<0.02$, the data were considered highly significant.

\section{RESULTS}

\section{Subjective Health Assessment}

The two questionnaires, although qualitative in nature, showed some interesting trends. The open question that was asked to each participant at the end of the 5-weeks consumption period was: "Did you feel any changes in your health that you believe were related to consuming the capsules? If so, please describe." All 6 females and 6 males in the placebo group commented that they had not noticed any changes. One female in the EpiCor ${ }^{\circledR}$ group commented on increased energy and accelerated recovery after a flu episode.

Most interestingly, the allergy season started during the 5 -week consumption period, and 3 out of 6 males in the EpiCor $^{\circledR}$ group noticed a complete absence of seasonal allergies when consuming EpiCor ${ }^{\circledR}$, with a full return of allergies within 1-2 weeks after stopping the consumption of EpiCor ${ }^{\circledR}$. When analyzing the second questionnaire with 22 questions pertaining to minor health complaints, participants who consumed EpiCor ${ }^{\circledR}$ had fewer health complaints at the end of the study $(p<0.05)$, whereas the placebo group showed no changes. The reduction in health complaints after consumption of EpiCor ${ }^{\circledR}$ compared to placebo was highly significant $(p<0.02)$.

\section{Complete Blood Count (CBC)}

The CBC with differential was performed in triplicate on all blood samples. The health of erythrocytes was evaluated by erythrocyte count, hemoglobin, and hematocrit. EpiCor ${ }^{(B)}$ consumption did not result in an increase in RBC numbers, but led to an increase in hemoglobin and hematocrit Table 2. The increase in hematocrit was statistically significant $(p<0.04)$.

The CBC data regarding leukocyte counts showed changes upon consumption of EpiCor ${ }^{\circledR}$ versus placebo. The total white blood cell count remained constant in the EpiCor $^{\circledR}$ group, whereas there was a mild trend towards an increase in white blood cells in the placebo group $(p<0.25$, Fig. (1A)), likely due to the onset of seasonal allergies in people in the placebo group. The placebo group showed an overall increase in granulocyte count over the five weeks of consumption $(p<0.03)$, whereas the EpiCor ${ }^{\circledR}$ group showed sumption $(p<0.03)$, whereas the EpiCor ${ }^{\circledR}$ group showed only a minor increase $\left(p<0.15\right.$, Fig. (1B)). Both the EpiCor ${ }^{\circledR}$ and placebo groups showed a mild decrease in lymphocyte counts after consumption; however, this was not significant (Fig. 1C).
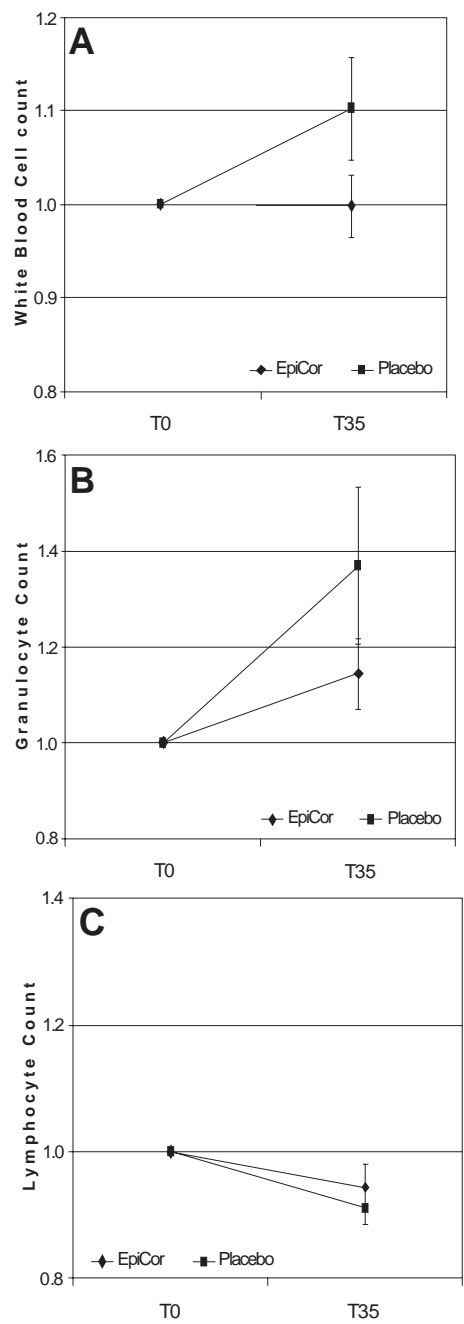

Fig. (1). Changes in white blood cells and subsets after 5 weeks consumption of either Epicor ${ }^{B}$ or placebo. A. Levels of white blood cells remained constant in the Epicor ${ }^{\circledR}$ group but increased in the placebo group, reflecting the onset of seasonal allergies. The difference between Epicor ${ }^{(B)}$ and placebo was not statistically significant $(p=0.27)$. B. A mild increase in granulocytes was seen in the Epi$\operatorname{cor}^{\mathbb{B}}$ group, contrasted by a larger increase in the placebo group $(p<0.2)$. C. A minor decrease in lymphocytes counts were seen in both groups. 


\section{Saliva and Serum Immunoglobulins}

Due to the individual variations in immunoglobulin levels, analysis on the absolute concentrations of immunoglobulin between subjects in each group showed no significant trend.

However, when we examined the normalized data, i.e. the change in each individual, the following observations emerged. When analyzing saliva secretory IgA ( $\operatorname{IgA}$ ) between the two groups, the EpiCor ${ }^{\mathbb{B}}$ group showed a relative increase in sIgA after consumption, whereas the placebo group showed a relative decrease in saliva IgA. The mean+/SD for each group and time point is shown in Fig. (2A). The difference did not reach statistical significance over the 5 week consumption period, but there was a visible trend $(p=0.16)$.

In the subsequent open label study involving 22 people consuming EpiCor ${ }^{\mathbb{B}}$ for 8 weeks, the increase in saliva sIgA did not reach statistical significance after 4 weeks $(p<0.09)$, but did after 8 weeks EpiCor ${ }^{\circledR}$ consumption $(p<0.05)$.

Serum IgA also showed a mild increase, but the trend was much milder than saliva sIgA, and not significant (Fig. 2B). Serum IgG increased over the 5-week consumption period in both groups (Fig. 2C). The trend was strongest in the EpiCor ${ }^{\circledR}$ group, but the difference between EpiCor ${ }^{\circledR}$ and placebo did not reach statistical significance.

The individual levels of serum IgE levels in the placebo group escalated during the 5 weeks $(p<0.13)$, whereas the serum IgE levels only showed a minor increase in the Epi$\mathrm{Cor}^{\mathbb{R}}$ group during this time. The 5 -week consumption period fell at the time where seasonal allergies started. Serum IgE measurements were not originally planned as part of the study, but were performed after the end of the study, after we received feedback from some of the study participants in the EpiCor $^{\mathbb{B}}$ group regarding the unexpected absence of their usual seasonal allergies. A systematic evaluation of the occurrence and severity of seasonal allergies was not performed, but based on the information gathered in this study; a larger human clinical trial is currently being conducted, specifically addressing this question. There was a trend showing higher serum IgE in the placebo group than in the EpiCor $^{\mathrm{B}}$ group after the 5-week consumption period. Despite the trend, the difference in the individual variations in serum $\operatorname{IgE}$ were not statistically significant between the EpiCor ${ }^{\circledR}$ and placebo group (Fig. 2D).

\section{C-Reactive Protein (CRP)}

Based on the high ORAC value of EpiCor ${ }^{\circledR}$ and the antiinflammatory effects seen in cell-based assays [3], it was of interest to examine whether EpiCor ${ }^{\mathbb{B}}$ consumption would have an effect on a marker of inflammation. We chose to examine serum $\mathrm{C}$-reactive protein (CRP), even though we were examining minor changes well within the normal range (i.e. below $6 \mathrm{mg} / \mathrm{L}$ ) and not associated with any pathology. The individual CRP levels remained constant in study participants in the placebo group, and in most of the participants in the EpiCor ${ }^{B}$ group. Two people in each group had a higher starting level of CRP than the other participants, above $0.6 \mathrm{mg} / \mathrm{L}$. This higher level of CRP did not change in the two people from the placebo group, but was reduced to below $0.4 \mathrm{mg} / \mathrm{L}$ in the EpiCor ${ }^{\mathbb{R}}$ group (Fig. 3).
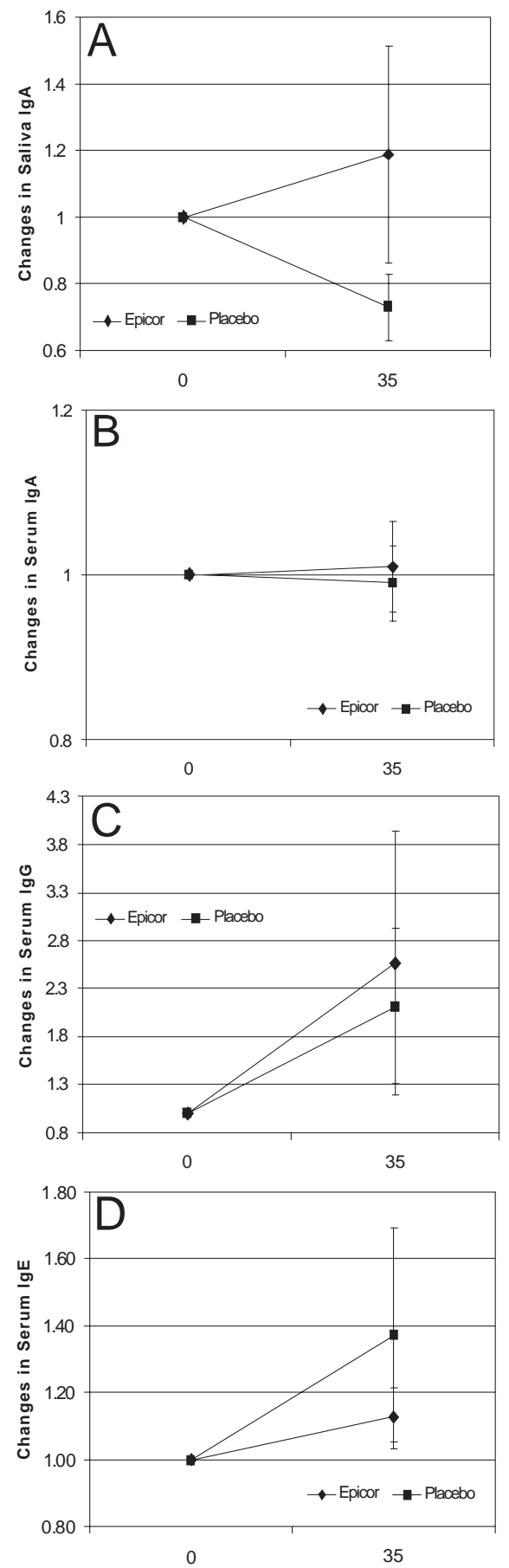

Fig. (2). Changes in saliva and serum immunoglobulins after 5 weeks consumption of either Epicor ${ }^{\circledR}$ or placebo. A. Saliva IgA increased after Epicor ${ }^{\mathbb{B}}$ consumption but decreased after placebo consumption $(p=0.16)$. B. Serum $\operatorname{IgA}$ showed the same trend, but the difference was minor and not statistically significant. C. Serum IgG increased in both groups, however it increased much more in the placebo group. D. Serum IgE remained almost constant in the Epicor $^{\mathbb{B}}$ group whereas it increased in the placebo group, however, the difference was not statistically significant.

\section{Th1/Th2 Cytokine Profile in Serum}

As a further assessment of inflammatory status of the study participants, we evaluated the Th1/Th2 cytokine profile in serum samples. This profile includes the 6 cytokines 
IL-2, IL-4, IL-6, IL-10, TNF- $\alpha$, and IFN- $\gamma$. When analyzing individual changes in cytokine levels, no significant differences were observed between the two groups for IL-2, Il-4, IL-6, TNF-a, or IFN-g. However, the individual IL-10 levels were increased after EpiCor ${ }^{\circledR}$ consumption, while they remained unchanged in the placebo group (Fig. 4). The difference in changes in IL-10 levels between the two groups was indicative of a possible trend towards induction of IL-10 by $\operatorname{EpiCor}^{\circledR}(p<0.2)$.

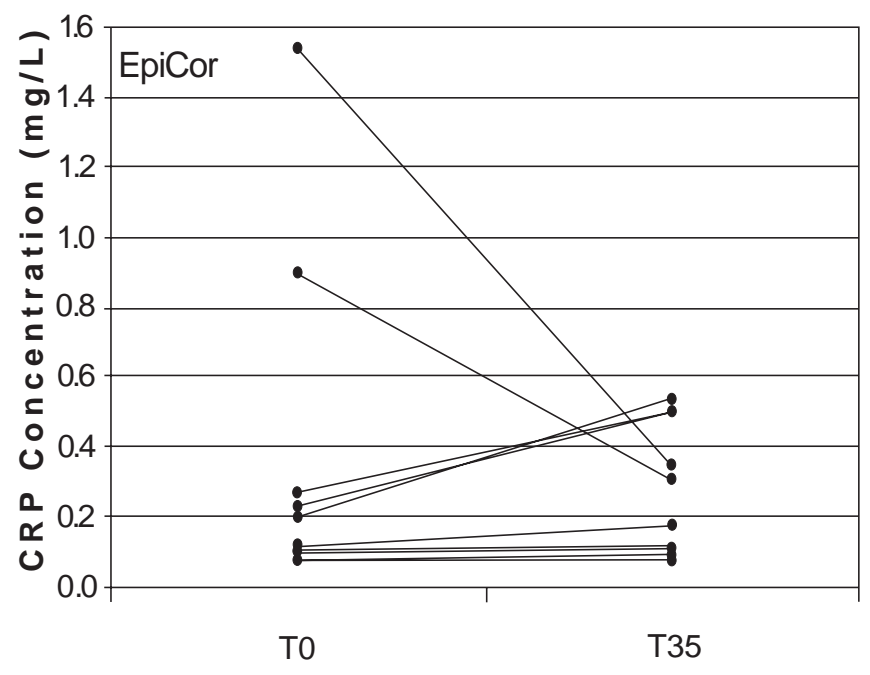

Fig. (3). Individual levels of serum levels of C-reactive protein (CRP) before and after 5 weeks of consumption of Epicor ${ }^{\circledR}$ or placebo. In each group, two individuals had CRP levels in the higher end of the normal range, i.e. above $0.6 \mathrm{mg} / \mathrm{L}$. The CRP levels decreased to below $0.4 \mathrm{mg}$.L in the two individuals consuming Epi$\operatorname{cor}^{\circledR}$, whereas the CRP levels remained unchanged in the two individual s consuming placebo.

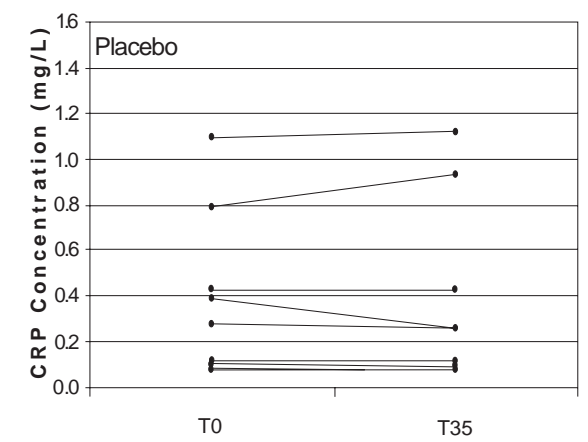

Fig. (4). Individual levels of IL-10 before and after 5 weeks of consumption of EpiCor ${ }^{\circledR}$ or placebo. The IL-10 levels in serum remained unchanged in the placebo group. In contrast, an increase was seen in the EpiCor ${ }^{\circledR}$ group $(p=0.2)$.

\section{Effects of EpiCor ${ }^{\mathrm{TM}}$ on B Lymphocytes In Vitro}

Given that EpiCor ${ }^{\circledR}$ enhanced the production of $\operatorname{SIgA}$ in vivo, the concern arose whether EpiCor ${ }^{\circledR}$ could possibly have an undesired, supportive effect on the growth of leukemia B cells. This was not the case. We found that at doses used for our previously published in vitro work on peripheral blood $\mathrm{B}, \mathrm{T}$, and NK cells [3], there was a highly significant growth inhibition when the Daudi B leukemic B cell line was treated with EpiCor ${ }^{\circledR}$ at doses up to $10 \mathrm{mg} / \mathrm{L}$ (Fig. 5).

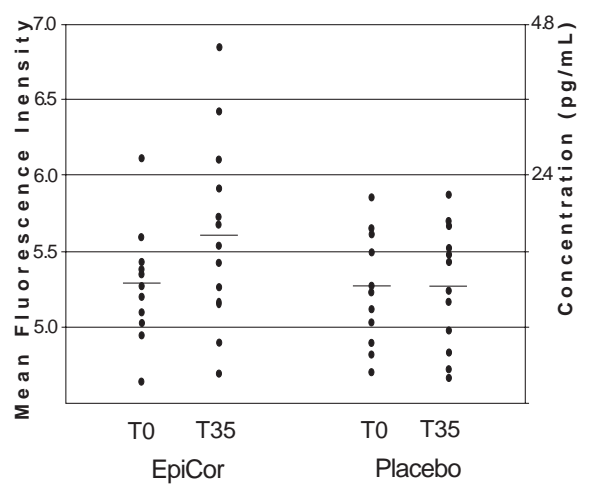

Fig. (5). The growth of the Daudi leukemia cell line was inhibited by EpiCor ${ }^{\circledR}$ at doses higher than $0.4 \mathrm{mg} / \mathrm{L}$. At the dose of $10 \mathrm{mg} / \mathrm{L}$ there was an almost $90 \%$ inhibition of cell growth in the Daudi cell cultures. Untreated cultures were performed in quadruplicate and each serial dilution of EpiCor ${ }^{\circledR}$ was tested in duplicate. Mean $+/-$ standard deviation are shown. The data shown are representative of three similar experiments.

We decided to perform further testing on effects of survival of healthy versus leukemia $B$ cells at higher doses. We conducted testing at very high doses that were creating disturbances in the culture medium for healthy B cells. Under those extreme conditions, healthy B cells were more tolerant to EpiCor ${ }^{\mathbb{R}}$ than the Daudi leukemia cells (Fig. 6).

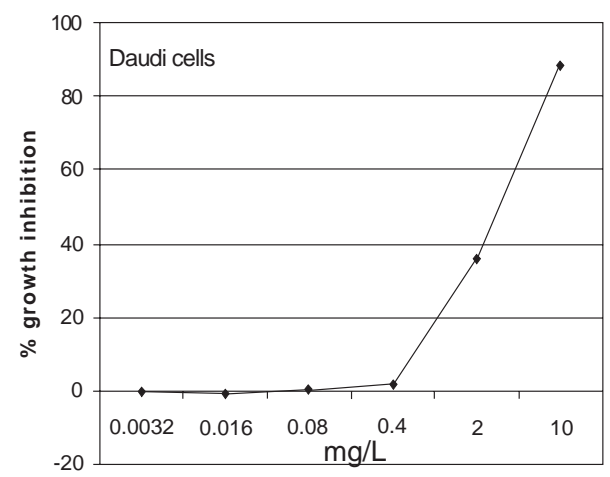

Fig. (6). Effects of very high doses of Epicor ${ }^{\circledR}$ on induction of apoptotic cell death the Daudi B lymphoblastic tumor cell line in comparison to PBMC B lymphocytes in vitro. The \% B cells undergoing advanced apoptosis, as evaluated by co-expression for both Annexin V (AVX) and 7AAD, was evaluated in cultures of peripheral blood mononuclear cells (PBMC) and the Daudi leukemia cell line. At doses lower than $10 \mathrm{mg} / \mathrm{L}$ there was no effect on cell death (data not shown). At doses between 25 and $50 \mathrm{mg} / \mathrm{L}$, the Daudi leukemia cells were less tolerant to EpiCor ${ }^{\circledR}$ treatment than PBMC $(p<0.005)$. Epicor ${ }^{\mathbb{B}}$ doses higher than $100 \mathrm{mg} / \mathrm{L}$ in the culture medium were too high for both cell types.

\section{DISCUSSION}

Methods for improving general health by reducing inflammation and providing support for a healthy immune function are receiving intense interest from many fields of research, including the evaluation of nutritional and antiinflammatory nutraceutical strategies. 
The data presented here were based in an exploratory study design, aimed at identifying parameters in healthy adult human subjects that would show trends towards changes after EpiCor ${ }^{\mathbb{R}}$ consumption. It was encouraging that several trends were identified despite the small study population, including parameters that were statically significant. The analysis of the subjective answers regarding minor health complaints showed a significant reduction in complaints after 5 weeks of EpiCor ${ }^{\mathbb{R}}$ consumption $(p<0.02)$.

The consumption of Epicor ${ }^{\circledR}$ resulted in a significant increase in hematocrit $(p<0.04)$, suggesting a beneficial effect on the marrow environment and red blood cell production. An increase in hemoglobin was also observed but did not reach statistical significance. We conclude that the consumption of EpiCor ${ }^{\circledR}$ resulted in production of healthier RBC, since their numbers did not change, but their volume (as measured by the hematocrit) and content of hemoglobin increased compared to baseline. Improvement of marrow function and resulting increase in red blood cell production was reported for Spirulina in a mouse model [10]. Consumption of another microbial-based nutritional supplement has been shown to provoke a rapid mobilization of stem cells from marrow in humans, most likely by affecting the bone marrow environment [11]. Adult stem cells are involved in many types of repair including natural processes of tissue remodeling and maintenance of integrity in muscle tissue, central nervous system, liver, kidney and many other tissues. Support of various aspects of stem cell biology has impact on many aspects of health, including prevention of heart disease, diabetes, and cognitive decline. Thus, nutritional strategies for improvement of marrow function and natural repair and rejuvenation mechanisms are subject for serious research efforts.

The timing of the study was such that seasonal allergies started shortly after the study commenced, and we believe that this is an important factor for understanding these data. This data should be seen in the context of the subjective comments, where several participants in the EpiCor ${ }^{\mathbb{B}}$ group reported an absence of their usual seasonal allergies, and some reported that allergies appeared after consumption of EpiCor $^{B}$ was stopped. It is therefore possible that EpiCor ${ }^{B}$ was protective against allergies, at least in some participants. The testing of IgE was added after completion of the clinical study, based on this feedback. Hence, systematic information on the onset of seasonal allergies was not collected, as we had not anticipated this effect. A placebo-controlled clinical trial is currently ongoing to specifically address this question in a larger group of people.

Based on the EpiCor ${ }^{\circledR}$-mediated specific activation of B lymphocytes seen in vitro [3], as well as the previously reported increase in salivary secretory $\operatorname{IgA}$ in factory workers exposed to a similar product [2], it was an important part of this in vivo study to examine, even at a pilot level, to what extent EpiCor ${ }^{\mathbb{B}}$ consumption modulated the production of various immunoglobulins. Saliva IgA is the immunoglobulin which protects mucosal surfaces from microbial invaders. A mild increase in saliva IgA was observed in the EpiCor ${ }^{\mathbb{B}}$ group, in contrast to a mild decrease in the placebo group. While the data did not reach statistical significance, it suggested that EpiCor ${ }^{\circledR}$ consumption supports immune protection across mucosal surfaces. Additionally, a longer open label trial ( 8 weeks) did reach statistical significance for increased saliva $\operatorname{sIgA}(p<0.05)$.

The decrease in saliva $\operatorname{sIgA}$ seen in the placebo group may be explained in part by the higher level of serum IgE suggesting that overall the participants in the placebo group were experiencing the onset of seasonal allergies. A link between allergic rhinitis and reduced $\operatorname{sIgA}$ has been proposed by another study [12].

CRP is increasingly used to monitor differences, even within the high-end of the normal range as a measure of wellness [13], and is linked as a risk factor in cardiovascular disease [14]. The overall state of inflammation was marginally higher in the placebo group than in the Epicor ${ }^{\circledR}$ group after the 5 week consumption ended.

The consumption of EpiCor ${ }^{\circledR}$ did not alter Th1/Th2 cytokine profile, with the interesting exception of IL-10. The serum level of IL-10 was mildly up-regulated upon EpiCor ${ }^{\circledR}$ consumption $(p=0.2)$, which not only supports an overall anti-inflammatory role of EpiCor ${ }^{\mathbb{B}}$, but also suggests an effect on T-regulatory cells, known to control immune responses via IL-10 production $[15,16]$. To what extent consumption of an immunogen initiates events that may partially overlap with immune reactions initiated by mucosal immunization strategies is unknown. However, mucosal introduction of immune activating compounds, including sublingual vaccination, has been shown to induce IL-10 production as part of a regulatory role [17].

Based on the strong NK activation seen in vitro when healthy lymphocytes were exposed to EpiCor ${ }^{B}$, there could be a concern regarding whether the daily consumption of such a strong immunomodulatory compound would overstimulate the human immune system, leading either to unsafe levels of inflammatory cytokines, increased levels of white blood cells, or over-stimulation of antibody production. None of these concerns were supported by our data.

As an overall conclusion to this pilot study, the data suggest that EpiCor ${ }^{\mathbb{B}}$ supports the formation of healthy red blood cells, supports mucosal defense and provides antiinflammatory support. Future studies on chronically inflamed individuals such as patients suffering from chronic fatigue syndrome or cardiovascular disease, are warranted.

\section{ACKNOWLEDGMENTS}

We thank Anna Gawlicka. PhD, AIBMR Life Sciences, for initial help with study design. We thank Lue Ann Zaske, Charlene Mogle R.N., Sharon Bush R.N., Kimberlee A. Redman, Aaron N. Hart, BSc, Tracy Werner and Dave Severson for excellent technical assistance. The study was sponsored by Embria Health Sciences, 2105 SE Creekniew Drive, Ankeny, IA 50021.

Note: S.G. Reeves is the director for research and development, and L.E. Robinson is the vice president for scientific affairs for Embria Health Sciences, the manufacturer of Epi$\mathrm{Cor}^{\circledR}$. No other authors have any financial interest in the subject matter.

\section{REFERENCES}

[1] Schauss AG. Suplemento alimentar unico que proporciona a melhoria imunologica. [A novel yeast fermentate.]. Rev Bioquim Med $2006 ; 15(3): 30-32$. 
[2] Schauss AG, Vojdani A. A discovery of an edible fermentation product with unusual immune enhancing properties in humans. FASEB J 2006; 20: A143.

[3] Jensen GS, Hart AN, Schauss AG. An anti-inflammatory immunogen from yeast culture induces activation and alters chemokine receptor expression on human natural killer cells and B lymphocytes in vitro. Nutr Res 2007; 27: 327-35.

[4] Bastard JP, Maachi M, Lagathu C, et al. Recent advances in the relationship between obesity, inflammation, and insulin resistance. Eur Cytokine Netw 2006; 17: 4-12.

[5] Lau FC, Shukitt-Hale B, Joseph JA. Nutritional intervention in brain aging: reducing the effects of inflammation and oxidative stress. Subcell Biochem 2007; 42: 299-318.

[6] Dröge W, Schipper HM. Oxidative stress and aberrant signaling in aging and cognitive decline. Aging Cell 2007; 6: 361-70.

[7] Li Y, Zhang J. Serum concentrations of antioxidant vitamins and carotenoids are low in individuals with a history of attempted suicide. Nutr Neurosci 2007; 10: 51-8.

[8] Takahashi T, Yu F, Zhu SJ, et al. Beneficial effect of brewers' yeast extract on daily activity in a murine model of chronic fatigue syndrome. Evid Based Complement Alternat Med 2006; 3: 109-15.

[9] Li J, Li DF, Xing JJ, Cheng ZB, Lai CH. Effects of beta-glucan extracted from Saccharomyces cerevisiae on growth performance, and immunological and somatotropic responses of pigs challenged with Escherichia coli lipopolysaccharide. J Anim Sci 2006; 84: 2374-81.
[10] Zhang Cheng-Wu. Effects of polysaccharide and phycocyanin from spirulina on peripheral blood and hematopoietic system of bone marrow in mice. Nanjing Univ. China. Published in Proc. of Second Asia Pacific Conf. on Algal Biotech. Univ. of Malaysia. April 1994. p. 58.

[11] Jensen GS, Hart AN, Zaske LAM, et al. Mobilization of human CD34+CD133+ and CD34+CD133- stem cells in vivo by consumption of an extract from Aphanizomenon flos aquae - Related to modulation of CXCR4 expression by an L-selectin ligand? Cardiovasc Revasc Med 2007; 8: 189-202.

[12] Paparo BS, Leri O, Andreoli P, et al. Allergic rhinitis, olfactory disorders and secretory IgA. Riv Eur Sci Med Farmacol 1996; 18(4): 157-61.

[13] Kao PC, Shiesh SC, Wu TJ. Serum C-reactive protein as a marker for wellness assessment. Ann Clin Lab Sci 2006; 36: 163-9.

[14] Gotto AM Jr. Role of C-reactive protein in coronary risk reduction: focus on primary prevention. Am J Cardiol 2007; 99: 718-25.

[15] Goetzl EJ. Changing paradigms in the immunological science of allergy. Curr Allergy Asthma Rep 2007; 7: 7-10.

[16] O'Garra A, Vieira P. Th1 cells control themselves by producing Interleukin-10. Nat Rev Immunol 2007; 7: 425-428.

[17] Ciprandi G, Fenoglio D, Cirillo I, et al. Sublingual immunotherapy: an update on immunologic and functional effects. Allergy Asthma Proc 2007; 28: 40-3.

C) Jensen et al:; Licensee Bentham Open.

This is an open access article distributed under the terms of the Creative Commons Attribution License (http://creativecommons.org/licenses/by/2.5/), which permits unrestrictive use, distribution, and reproduction in any medium, provided the original work is properly cited. 